\title{
Valores culturais, reputação e governança das empresas preferidas pelas mulheres brasileiras
}

\section{Cultural Values, Reputation and Corporate Governance of Organizations Preferred by Brazilian Women}

\author{
DANIVAL SOUSA CAVALCANTE* \\ IGOR RODRIGO MENEZES TEODÓSIO** \\ MÁRCIA MARTINS MENDES DE LUCA***
}

\section{RESUMO}

Considerando que cada empresa traz consigo, explícita ou implicitamente, seus valores culturais, que podem atrair as mulheres para seu ambiente de trabalho, este estudo tem o objetivo de analisar os valores culturais organizacionais adotados pelas empresas preferidas pelas mulheres brasileiras e suas relações com a reputação e a governança corporativa. Adicionalmente, verifica-se possíveis associações entre os valores culturais e as características das empresas, comparando-se os valores culturais entre as empresas com reputação corporativa e as demais, e entre aquelas que adotam princípios de governança corporativa e as demais. $\mathrm{O}$ trabalho diferencia-se por analisar, numa mesma investigação, os construtos valores culturais, reputação e governança corporativas. Além da análise qualitativa das informações, o estudo utiliza técnicas de estatística descritiva e inferencial para a análise quantitativa dos dados. Dentre as empresas preferidas pelas mulheres brasileiras, a dimensão cultural respeito, com seu valor cultural desenvolvimento, foi a mais presente nas empresas investigadas. Verificou-se que as empresas adeptas de princípios de governança corporativa registram uma quantidade de valores culturais superior à das demais, assim como foi pos-

\footnotetext{
* Universidade Federal do Ceará. danivalsc@hotmail.com

** Universidade Federal do Ceará. igor.teodosio@hotmail.com

*** Universidade Federal do Ceará. marciammdeluca@gmail.com
} 
sível identificar associação entre as variáveis valores culturais e governança corporativa.

Palavras-chave: Valores culturais; Governança corporativa; Reputação corporativa; Mulheres no ambiente corporativo.

\section{Abstract}

Each firm is explicitly or implicitly informed by a set of cultural values. Work environments generated by such values may or may not be attractive to female professionals. In this study, we looked at the cultural values adopted by organizations preferred by Brazilian women and tested their correlation with reputation and corporate governance. We also probed for associations between cultural values and company profile by dividing the sample into firms with and without corporate reputation and firms with and without corporate governance. The study is unique in that it analyzes the constructs "cultural values', 'reputation' and 'corporate governance' in a single investigation. Qualitative and quantitative data were collected and analyzed, the latter with descriptive and inferential statistics. The cultural dimension 'respect' (and, within it, the cultural value 'development') was predominant in the sample. The number of cultural values was greater among adopters than non-adopters of corporate governance, and a correlation was observed between 'cultural values' and 'corporate governance'.

Keywords: Cultural values; Corporate governance; Corporate reputation; Women in the corporate world.

\section{INTRODUÇÃo}

A cultura organizacional constitui uma das várias formas utilizadas pelas empresas para revelar sua identidade corporativa e, por consequência, sua imagem para os stakeholders. O tema tem sido objeto de interesse de inúmeros pesquisadores, que procuram relacioná-lo com diferentes construtos, como o desempenho empresarial, a criação de valor para os acionistas, a reputação corporativa e a governança corporativa.

Fleury e Fischer (1989, p. 22) definem a cultura em um ambiente corporativo como "um conjunto de valores e pressupostos básicos expressos em elementos simbólicos", que tem a capacidade de ordenar, atribuir significações e ajudar a constituir a identidade 
organizacional, que tanto age como elemento de comunicação e consenso, como oculta e instrumentaliza as relações de dominação. Nesse contexto, a cultura se relaciona com a identidade corporativa, que, por sua vez, relaciona-se com a reputação corporativa.

Greyser (1999) avalia que a cultura organizacional pode influenciar a reputação corporativa, particularmente em função das expectativas públicas, já que a reputação constitui uma representação perceptual das ações passadas e das perspectivas futuras que descrevem a percepção global da empresa para os stakeholders. Essa percepção global da empresa também é afetada pelo ambiente confiável e seguro, característico daquelas instituições que adotam princípios de governança corporativa (NARDI; NAKAO, 2008).

Casavechia, Medeiros e Valadão Júnior (2011) argumentam que a maioria dos estudos que investigam os elementos da cultura corporativa se concentram na teoria de perspectiva de integração e evidenciam uma forte busca pelo consenso nas manifestações culturais, com ações continuadas para a integração dos valores e crenças apregoados pelas organizações, podendo influenciar a imagem corporativa na sociedade.

O presente estudo parte da percepção e das preferências das mulheres sobre as empresas onde trabalham, a partir do ranking dos 20 empregadores mais bem avaliados pelas mulheres brasileiras em 2017, divulgado pela comunidade de carreiras Love Mondays (2017), que ouviu mais de 25 mil mulheres profissionais sobre os seus empregadores preferidos. $\mathrm{O}$ resultado dessa pesquisa mostra que a satisfação das mulheres com a carreira aumentou entre 2016 e 2017, sendo a cultura organizacional o fator que mais trouxe satisfação para as profissionais brasileiras (LOVE MONDAYS, 2017).

Destarte, como cada empresa traz consigo, explícita ou implicitamente, seus valores culturais, que podem atrair ou não a mulher para seu ambiente de trabalho, este estudo pretende responder ao seguinte questionamento: quais os valores culturais adotados pelas empresas preferidas pelas mulheres profissionais no Brasil e como eles se relacionam com a reputação corporativa e a governança corporativa?

Diante da questão ora formulada, o estudo tem como objetivo geral analisar os valores culturais organizacionais adotados pelas empresas preferidas pelas mulheres brasileiras e suas relações 
com a reputação e a governança dessas empresas. Adicionalmente, compara-se os valores culturais entre as empresas com reputação corporativa e as demais, e entre aquelas que adotam princípios de governança corporativa e as demais.

Conforme explicam Silva Júnior, Junqueira e Bertucci (2009), diversos estudos buscam relacionar a governança corporativa com variáveis quantitativas, econômico-financeiras, buscando encontrar efeitos, influências, dentre outras. Contudo, diferente desses estudos, esta pesquisa busca relacioná-la à variáveis qualitativas como os valores culturais e a reputação corporativa, examinando possíveis associações entre elas.

Faz-se aqui, portanto, uma análise qualitativa dos dados coletados, especialmente no que tange aos valores culturais divulgados pelas empresas, assim como se utilizam técnicas de estatística descritiva e inferencial, fundamentando-se as comparações e correlações propostas.

O estudo contribui para ampliar a literatura científica sobre cultura organizacional e justifica-se por discutir sobre a participação das mulheres no seio corporativo, historicamente dominado pelos homens (NASCIMENTO, 2016), bem como por ressaltar a percepção das mulheres no ambiente organizacional. Na prática, o debate interessa aos gestores que buscam maior produtividade e um melhor ambiente de trabalho, na medida em que aponta os valores culturais que mais satisfazem as profissionais brasileiras. Além disso, diferencia-se por analisar, conjuntamente, os construtos cultura organizacional, reputação e governança corporativa, considerando que não foram encontradas, nas principais bases de periódicos nacionais e internacionais, investigações que abordam esses temas em um único estudo.

\section{REVISÃo DE LITERATURA}

\subsection{Cultura organizacional, reputação corporativa e gover- nança corporativa}

A cultura organizacional está associada aos valores, crenças, costumes e perfis dos administradores, ou mesmo dos proprietários, considerando-se a natureza da empresa (se familiar ou não), a for- 
ma jurídica, o porte, o segmento econômico, sua história, além de outros fatores (FLEURY, 1987; FREITAS, 1991; PETTIGREW, 1979).

Cameron e Quinn (2006, p. 31) definem a cultura organizacional como "um conjunto de valores fundamentais, suposições, interpretações e abordagens que caracterizam a organização, sendo que cada perfil cultural pode afetar, de forma distinta, o seu sucesso empresarial".

Flamholtz e Randle (2012) consideram que existem vários níveis de cultura em uma organização. Dentre os diferentes níveis, os autores destacam o nível que não está manifesto, mas implícito tanto no nível superficial, que é observável na organização, e central, normas e diretrizes comportamentais, no qual há um conjunto de atributos culturais que é considerado o "DNA" da cultura e que revela a personalidade corporativa, refletindo diretamente no comportamento das pessoas dentro das organizações (HARTNELL; OU; KINICK, 2011; PAULA et al., 2011; TOMÉ; VIEIRA, 2017).

É importante destacar o papel dos valores culturais como elemento fundamental da cultura organizacional. Nunes, Tolfo e Garcia (2018, p. 125) definem os valores organizacionais como "princípios organizados em ordem de importância, referindo-se ao que é central e ao que é periférico no contexto organizacional, ou ao que é desejável ou indesejável para a entidade". Demo, Fernandes e Fogaça (2017) afirmam que os valores culturais têm o objetivo de orientar a organização e guiar comportamentos que são fundamentais no cotidiano das empresas.

Segundo Pires e Macedo (2006), cada organização possui suas peculiaridades e reflete os valores culturais da sociedade maior. Tais valores culturais são transmitidos para as pessoas através do processo de socialização e são consolidados com sua prática social no cotidiano das instituições (PIRES; MACEDO, 2006; VIEIRA, 2007). Importa ressaltar que a cultura organizacional, quando se configura a partir de valores associados à atuação da liderança, favorece a existência de um ambiente propício ao compartilhamento do conhecimento (VASCONCELOS et al., 2013), gerando maior competitividade e proporciona a harmonização dos interesses dos diferentes stakeholders.

Neste estudo, representada pelos valores culturais, a cultura organizacional se estende às atividades empresariais, que pode re- 
sultar em uma reputação conquistada perante a sociedade, capaz de gerar vantagem competitiva sustentável e melhorar o desempenho empresarial (FLAMHOLTZ; RANDLE, 2012; FLATT; KOWALCZYK, 2008). Nesse contexto, alguns estudiosos apontam ainda que a cultura organizacional se destaca dentre os diversos fatores que antecedem a conquista da reputação corporativa (FLATT; KOWALCZYK, 2008; ROBERTS; DOWLING, 2002).

Guiso, Sapienza e Zingales (2015) explicam que há uma indicação da existência da relação de influência dos valores culturais na comunicação corporativa, tornando visível que uma base consolidada de valores culturais venha a incentivar a comunicação da empresa com os stakeholders, na obtenção de legitimidade perante estes, maior competitividade no mercado e melhor imagem corporativa.

Na visão de Chun (2005), a reputação corporativa reflete as impressões sobre a empresa apreendidas pelos agentes internos e externos que com ela se relacionam. Rindova, Williamson e Petkova (2005) complementam afirmando que a percepção dos stakeholders sobre a capacidade da organização pode criar valor diferenciado em relação aos seus concorrentes. Isso ocorre pelo fato da reputação corporativa possuir características que lhe conferem exclusividade, de modo que não pode ser replicada por outra empresa (DUTRA; PARENTE, 2018), tendo em vista que a reputação é algo insubstituível, pois não é algo que as empresas podem comprar ou vender, mas um recurso raro e inimitável, que lhes permite criar vantagem competitiva favorável ao desempenho financeiro (LEE; ROH, 2012).

Destaca-se ainda que a reputação corporativa pode ser considerada uma estratégia sustentável, que ajuda a empresa a alcançar vantagem em relação aos seus concorrentes, proporcionando desempenho superior e criação de valor (FLAMHOLTZ; RANDLE, 2012; MACEDO; CORRAR, 2012),

Pelo exposto, compreende-se que os sinais emitidos para o mercado, por meio da divulgação de informações e práticas da organização, podem ser convertidos em prestígio para a empresa, com base na percepção dos stakeholders. Nesse contexto, a reputação é consequência positiva de interações e experiências acumuladas entre a empresa e o mercado, proporcionando ascensão e credibilidade empresarial (FELDMAN; BAHAMONDE; BELLIDO, 2014). 
Para identificar melhor os seus efeitos no ambiente corporativo, a reputação deve estar, portanto, alinhada com outras variáveis empresariais, além da cultura organizacional, como, por exemplo, o desempenho econômico, o retorno de investimentos e as práticas de governança corporativa (BANDEIRA et al., 2015; CALEGARI et al., 2016).

Assim, as características e práticas de governança corporativa também podem representar sinais emitidos pelas empresas para o mercado, e, por consequência, afetar as percepções dos stakeholders sobre a organização (IBGC, 2015; LOPES; VALENTIN; FADEL, 2014). $\mathrm{O}$ aprimoramento das discussões sobre a governança no mundo corporativo se deu na busca de soluções para questões relacionadas às constatações de expropriação de riqueza de acionistas/investidores pelos gestores, apresentando o problema oriundo da Teoria da Agência (JENSEN; MECKLING, 1976).

Shleifer e Vishny (1997, p. 737) definem governança corporativa como um "conjunto de mecanismos por meio dos quais os investidores procuram assegurar o retorno dos seus investimentos", haja vista que, segundo Ferreira et al. (2019), esses mecanismos visam à minimização dos problemas e custos de agência (FERREIRA et al., 2019). Segundo Pound (1995), trata-se de mecanismo que propicia mais transparência, disciplina e responsabilidade para os administradores das empresas, em favor dos stakeholders, principalmente os acionistas. Lopes, Valentim e Fadel (2014) explicam-na como um modelo de gestão que visa a construir uma boa relação entre gestores, controladores, acionistas minoritários e demais atores.

Andrade e Rossetti (2009) destacam os princípios fundamentais da governança corporativa: a transparência (disclosure), a equidade (fairness), a prestação de contas (accountability) e a responsabilidade social (compliance). O primeiro deles está relacionado à evidenciação dos fatos, observando-se a salvaguarda dos interesses da empresa. A equidade propõe que a empresa observe os conflitos de interesses e procure solucioná-los, atendendo igualmente às partes. A prestação de contas conduz à apresentação de demonstrativos com qualidade, em que os stakeholders podem obter informações úteis para subsidiar suas decisões. E a responsabilidade social orienta as empresas a agir em conformidade com as normas. Minadeo (2019) 
ressalta que esse conjunto de elementos ajuda a orientar as diversas práticas de governança corporativa e são elementos fundamentais para o processo de decisão dos gestores.

A adoção dos princípios e, consequentemente, de melhores práticas de governança corporativa, cria um ambiente mais seguro para os acionistas e para os diversos stakeholders, sendo atribuídas credibilidade e confiabilidade à empresa (NARDI; NAKAO, 2008).

Verifica-se, assim, que as diversas definições de governança corporativa canalizam para um entendimento convergente, voltado para a aproximação entre gestores e acionistas, de modo a reduzir a assimetria informacional e, consequentemente, os custos e conflitos de agência, resultando em uma maior garantia de retorno do capital investido. Além disso, observa-se que, a partir da governança corporativa, estabelece-se um relacionamento mais transparente e responsável com todos os stakeholders.

Diante do exposto, e entendendo-se que a cultura organizacional precede a reputação corporativa (FLATT; KOWALCZYK, 2008; ROBERTS; DOWLING, 2002), e que esta, assim como a adoção dos princípios básicos de governança corporativa, emite sinais para a formação do conjunto de percepções internas e externas dos stakeholders sobre a empresa (CHUN, 2005; GREYSER, 1999; NARDI; NAKAO, 2008), espera-se que as variáveis reputação corporativa e governança corporativa estejam correlacionadas com a variável valores culturais, representativa da cultura organizacional, assim como é de se esperar que as empresas que possuam reputação e governança apresentem maior volume, quantitativo e proporcional de valores culturais.

\subsection{Presença feminina nas organizações}

Considerando-se que a motivação deste estudo teve origem na investigação sobre a preferência feminina no ambiente corporativo, importa apresentar algumas recentes pesquisas relacionadas à temática, a fim de contribuir para a fundamentação do presente debate e para melhor compreensão do atual estágio da participação feminina nas organizações empresariais.

Mills (1988) foi um dos precursores a relacionar a diversidade de gênero com a cultura organizacional, sob o argumento de que o gêne- 
ro é um aspecto crucial, mas negligenciado na análise organizacional. E complementa que a cultura organizacional fornece um foco crítico, mas não desenvolvido, para a análise de considerações de gênero.

Adams e Ferreira (2009) mostraram que as mulheres diretoras geram um impacto significativo sobre os insumos e resultados das empresas americanas; verificaram também que as diretoras têm melhores registros de atendimento do que os diretores; e observaram que as mulheres são mais propensas a se localizar em atividades de monitoramento. Jurkus, Park e Woodard (2011) investigaram a diversidade de gênero e seu efeito sobre os custos de agência das 500 maiores companhias listadas na Fortune e identificaram que as empresas com maior porcentagem de diretoras incorrem em menores custos de agência, mas que a correlação negativa não é robusta quando se considera a endogeneidade da diversidade, assim como a governança externa influencia o relacionamento.

Liu, Wei e Xie (2014) constataram nas empresas chinesas que as diretoras executivas produzem um efeito positivo mais forte no desempenho da empresa, se comparadas às diretoras independentes, indicando que o efeito-executivo supera o efeito-monitoramento. $\mathrm{Xu}$, Li e Chang (2016) verificaram, nas empresas listadas nas bolsas valores de Xangai e Shenzhen, que as organizações lideradas por Chiefs Financial Officers (CFOs) femininas são menos propensas a obter empréstimos do que aquelas lideradas por homens; e que os bancos exigem mais garantias quando emprestam para empresas lideradas por CFOs femininas, observando-se que estas enfrentam maior discriminação. Hoobler et al. (2016) investigaram a relação entre a representação das mulheres em cargos de liderança e o desempenho financeiro organizacional com base em 78 estudos realizados sobre a temática e verificaram que a presença de mulheres nos cargos de liderança está relacionada de forma positiva ao desempenho financeiro.

No Brasil, o estudo da temática também provoca relevante interesse entre os pesquisadores adeptos do assunto. Oliveira, Gaio e Bonacim (2009) identificaram uma tendência de maior valorização das mulheres, e que as organizações estão se dispondo a enfrentar as consequências desse novo contexto e ampliar o enfoque diretivo e o processo decisório para a inserção dos valores femininos, res- 
peitando a percepção das mulheres tanto nas questões sociais como naquelas de natureza organizacional.

Cavazotte, Oliveira e Miranda (2010) perceberam que a desigualdade tem impacto negativo nas atitudes das mulheres com relação às organizações que as empregam, e que, em consequência disso, a percepção de desigualdade afeta significativamente a intenção de se demitir. Nesse contexto, Ceribeli, Ceribeli e Ferreira (2016) constataram que as mulheres estão menos satisfeitas do que os homens em relação ao salário recebido, às oportunidades de crescimento profissional, aos critérios de recrutamento e seleção e à possibilidade de participação em comitês internos.

Menezes, Oliveira e Diniz (2013) identificaram alguns significados do trabalho executivo que seriam supostamente neutros quanto ao gênero, bem como das feminilidades associadas à (in) competência das mulheres para o trabalho executivo. A capacidade de negociação e organização, flexibilidade e cautela são apresentadas como ambivalentes, pesando ora a favor das mulheres que ocupam cargos de gestão, ora contra elas.

Salvagni e Canabarro (2015) verificaram que a construção e o sucesso na carreira implicam enfrentar o conflito do equilíbrio do pessoal com o profissional, e que elas se preocupam em desempenhar bem as várias facetas como mulheres, mães, esposas e gestoras, além de apresentar características de sensibilidade, flexibilidade e parceria no trabalho em equipe.

Oliveira et al. (2018) investigaram a relação entre o valor corporativo e a presença de mulheres na alta administração (conselho de administração e diretoria executiva) nas empresas brasileiras listadas na bolsa de valores oficial do Brasil, a Brasil Bolsa Balcão S/A (B3). Os resultados demonstraram uma relação positiva entre o valor da empresa e a presença de mulheres no conselho, evidenciando que a inclusão das mulheres no conselho, além de garantir a diversidade, atende aos preceitos da ética, da igualdade e da responsabilidade social, contribuindo para o melhor desempenho das empresas e gerando valor ao acionista.

Importa ressaltar que apesar da participação feminina na alta direção das organizações ser objeto de vários estudos, não foram encontradas pesquisas nas principais bases nacionais e interna- 
cionais, até a conclusão desse estudo, que relacionem ou analisem de forma conjunta a participação da mulher no ambiente corporativo, a importância dos valores culturais organizacionais como elementos atrativos para as mulheres no ambiente de trabalho, e os construtos reputação corporativa e governança corporativa, o que reforça a relevância desta pesquisa.

\section{Metodologia}

A partir do objetivo proposto o estudo se caracteriza como exploratório-descritivo. Além da análise qualitativa aplicada aos dados representativos dos valores culturais, a análise dos resultados toma por base técnicas de estatística descritiva e estatística inferencial.

A pesquisa abrange dados de uma população que, segundo Malhotra (2001), envolve a enumeração completa dos elementos de um grupo de interesse, que se torna viável quando seu número é pequeno. Assim, a população do presente estudo é constituída pelas organizações que integram o ranking dos 20 empregadores mais bem avaliados pelas mulheres brasileiras em 2017, desenvolvido e divulgado pela comunidade de carreiras Love Mondays (2017).

A comunidade de carreiras Love Mondays é uma startup, fundada no Brasil em 2014, cuja plataforma de avaliações de empresas resulta da participação de milhares de profissionais que avaliam as empresas onde trabalham de forma confidencial (LOVE MONDAYS, 2019). De acordo com a Love Mondays (2017), a pesquisa para a formação do ranking dos 20 empregadores mais bem avaliados pelas mulheres brasileiras em 2017 contou com a opinião de mais de 25 mil mulheres, a fim de descobrir os empregadores e setores por elas preferidos, com base em julgamentos anônimos compartilhados sobre as empresas e setores em que trabalham.

Assim, o presente estudo se propõe investigar os valores culturais organizacionais de todas as 20 empresas participantes do citado ranking; identificar os valores mais frequentemente adotados pelas organizações preferidas pelas profissionais brasileiras; analisar a possível associação entre os valores culturais e outras características observadas, como a presença feminina na alta administração, o setor econômico e a natureza jurídica; e analisar possíveis correlações 
com outras variáveis, como a prática de governança corporativa e a reputação corporativa, com o apoio de testes estatísticos.

Os dados para as análises do estudo são secundários e foram extraídos dos websites das empresas da amostra, em maio de 2017, compreendendo os seguintes conteúdos, no idioma português: apresentação, missão, visão, valores, compromisso, princípios, políticas e outras informações relacionadas aos valores culturais organizacionais. A identificação dos valores culturais, utilizou a proposta desenvolvida por Guiso, Sapienza e Zingales (2015), que, a partir da análise dos websites de diversas empresas, observaram que 85\% delas apresentam uma ou duas seções com informações sobre princípios e valores culturais. Destaca-se ainda que, na coleta dos dados, foram desconsiderados trechos duplicados e computados aqueles em que se reconheceu cruzamento dos construtos pesquisados.

Assim, para sistematizar e garantir uniformidade e objetividade à coleta dos dados, para a determinação da dimensão de cada valor cultural corporativo, seguiu-se a proposta de Guiso, Sapienza e Zingales (2015), segundo a qual uma dimensão (categoria) cultural específica pode ser representada por um conjunto de palavras-chave utilizadas pela empresa, destacando-se nove categorias culturais, a saber: integridade, trabalho em equipe, inovação, respeito, qualidade, segurança, comunidade, comunicação e trabalho pesado. A Figura 1 apresenta as palavras-chave representativas de cada uma das nove dimensões, propostas por Guiso, Sapienza e Zingales (2015), totalizando 62 possibilidades de palavras-chave que representam os valores culturais por empresa.

Assim, ao ser identificada no website a presença de alguma palavra-chave que represente uma dimensão, na língua portuguesa, conforme a Figura 1, atribuiu-se o valor " 1 "; e o valor " 0 ", se não foi localizada a palavra-chave para a dimensão cultural (GUISO; SAPIENZA; ZINGALES, 2015). A medida final de cada empresa para os valores culturais corporativos foi dada pelo somatório dos pesos correspondentes às palavras-chave componentes de cada dimensão cultural, podendo, teoricamente, variar de 0 a 62, dependendo do total de palavras-chave observadas para cada dimensão cultural. Nas análises estatísticas, foram considerados apenas os valores não repetidos por empresa; ou seja, considerou-se um único registro para cada palavra-chave. 


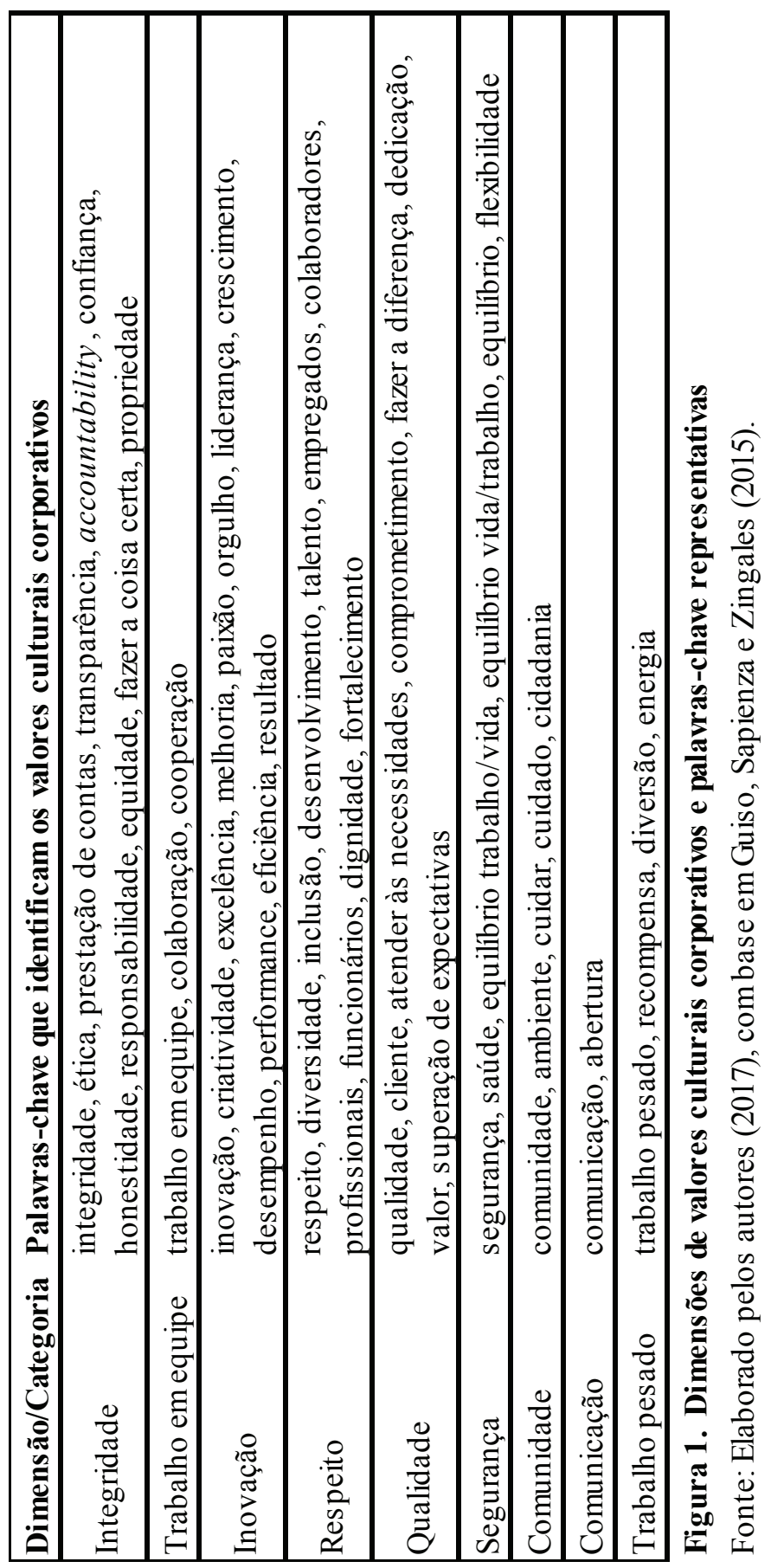


Para identificação de características empresariais complementares, a fim de se estabelecer possíveis comparações e associações entre estas e os valores culturais corporativos, foram considerados aspectos como a presença feminina na alta administração, o setor econômico, a natureza jurídica, a reputação corporativa e a governança corporativa.

A natureza jurídica e o setor econômico de cada empresa foram identificados mediante consulta ao Cadastro Nacional das Pessoas Jurídicas (CNPJ). A definição do setor econômico correspondente a cada empresa baseou-se na respectiva atividade econômica principal.

Para identificação da presença feminina na alta administração, investigaram-se informações e documentos disponíveis nos websites das empresas, abrangendo os cargos de presidência, vice-presidência e diretoria, atribuindo-se o valor 1 em caso positivo, e o valor nulo em caso negativo, funcionando como uma variável dummy.

Para a reputação corporativa, foi considerada a participação da empresa em um dos seis rankings reputacionais divulgados em 2016, a saber: Ranking Rep Trak® 100 no Brasil 2016 (Reputation Institute); As maiores companhias abertas no Brasil em 2016 (Revista Exame); As melhores empresas 2016 (revista Você S/A); Great Place to Work 2016 - Grandes (revista Época); Great Place to Work 2016 - Médias (revista Época); e 50 empresas mais amadas 2016 (Love Mondays). Nessa verificação, atribuiu-se o valor 1 a cada empresa presente em quaisquer desses rankings reputacionais, e valor nulo no caso contrário, funcionando como uma variável dummy.

Em relação à variável governança corporativa, consultaram-se, nos websites das empresas, informações que indiquem indícios de adoção de práticas de governança corporativa. Para sistematizar e uniformizar esse processo e garantir sua objetividade, foram observados os quatro princípios de governança corporativa estabelecidos pelo Código de Melhores Práticas de Governança Corporativa, do Instituto Brasileiro de Governança Corporativa (IBGC, 2015), a saber: transparência, equidade, accountability e responsabilidade corporativa. Contudo, para a operacionalização da variável governança corporativa, os princípios transparência, equidade e accountability não foram considerados na contagem, em virtude dessas mesmas palavras representarem também valores culturais corporativos, 
conforme proposta de Guiso, Sapienza e Zingales (2015) adotada no estudo, o que poderia causar viés nos resultados dos testes estatísticos. Dessa forma, as palavras-chave representativas da variável governança corporativa ficaram definidas em responsabilidade corporativa e o próprio termo governança corporativa. Atribuiu-se o valor 1 quando localizada uma dessas palavras-chave, e o valor nulo no caso contrário. Não foram consideradas as repetições de palavras-chave.

Após a fase de coleta e tabulação dos dados, procedeu-se o tratamento analítico qualitativo, seguido da análise quantitativa, com o uso de técnicas de estatística descritiva e inferencial. Devido ao pequeno número de elementos investigados, incialmente foi constatada a normalidade dos dados representativos dos valores culturais, por meio da aplicação do teste Shapiro-Wilk (S-W), tanto para a totalidade dos dados quanto para os dados segmentados por grupos de variáveis (cultura e reputação corporativa; e cultura e governança corporativa), justificando-se, assim, a aplicação de testes paramétricos, pois, segundo Fávero (2017), o teste S-W pode ser aplicado sobre populações de tamanho $4 \leq \mathrm{n} \leq 2.000$, como uma alternativa ao teste de normalidade de Kolmogorov-Smirnov (K-S) para pequenas amostras $(\mathrm{n}<30)$.

Destarte, comprovada a normalidade dos dados, foram realizados testes paramétricos de comparação entre médias independentes, para verificar as seguintes hipóteses: $\mathrm{H}_{1}$ - há diferença na quantidade de valores culturais entre as empresas com a presença de governança corporativa e as demais; e $\mathrm{H}_{2}$ - há diferença na quantidade de valores culturais entre as empresas com reputação corporativa e as demais.

Adicionalmente, a fim de se verificar possíveis associações entre as variáveis valores culturais, reputação corporativa e governança corporativa, foi aplicado o teste de correlação de Pearson, que, segundo Field (2009), serve para medir o relacionamento linear entre duas ou mais variáveis, e a intensidade desse possível relacionamento existente. Lira e Chaves Neto (2006) explicam ainda, que o teste fornece uma medida da relação entre uma variável numérica e outra variável com duas categorias ou dicotômicas, chamada de correlação ponto bisserial, como é o caso das variáveis de análise. 


\section{ANÁlise dos Resultados}

\subsection{Caracterização e análise descritiva dos dados das em- presas}

Foram submetidas a análise as 20 organizações brasileiras listadas no ranking dos empregadores mais bem avaliados pelas mulheres brasileiras em 2017, em pesquisa desenvolvida e divulgada pela comunidade de carreiras Love Mondays (2017), de acordo com a ordem crescente de classificação, a saber: Nubank, ClearSale, Monsanto, Rock Content, Takeda Brasil, Mercado Livre, Basf, Bayer, Porto Seguro, CIEE, Dr. Emerson Laboratório e Imagem, Braskem, Globosat, GSK, Amaggi, Mondelez, Dell, Odebrecht, Kimberly-Clark, e General Electric.

Observa-se que apenas duas empresas são de capital aberto (Braskem e Porto Seguro), uma é associação privada (CIEE), cinco são de capital fechado e doze são Sociedades Empresárias Limitadas. Em relação ao setor econômico principal observa-se a seguinte distribuição: três empresas de serviços de tecnologia, sete do setor de serviços diversos, sete do setor industrial, duas do setor comercial e uma do terceiro setor (associação privada).

Somente quatro das 20 empresas divulgaram a composição da alta administração, revelando-se a participação feminina em apenas duas delas: Amaggi (uma mulher dentre doze pessoas) e Odebrecht (duas mulheres dentre oito pessoas). Adams e Ferreira (2009) revelam que as agências Equal Opportunity for Women in the Workplace Agency (EOWA), em 2006, e European Professional Women's Network (EPWN), em 2004, identificaram apenas uma mulher, em média, no corpo de diretores das empresas.

Considerando-se os dados referentes aos valores culturais, e desconsideradas as repetições identificadas, foi observado um total de 238 palavras-chave distribuídas entre as empresas e as dimensões culturais. A Tabela 1 mostra a distribuição quantitativa das palavras-chave representativas de cada dimensão cultural, por empresa.

Embora seja a primeira na preferência das mulheres brasileiras segundo o ranking da Love Mondays (2017), a Nubank é uma das três empresas que menos se destacam na comparação relacionada aos valores culturais. Com efeito, essa empresa apresentou apenas 
Tabela 1 - Valores culturais por empresa e por dimensão

Empresa

Integri Trabalho Inova Res Quali Segu Comu Comuni Trabalho dade emequipe ção peito dade rança nidade cação pesado

\begin{tabular}{|c|c|c|c|c|c|c|c|c|c|c|}
\hline Nubank & 2 & - & - & - & 1 & - & - & - & - & 3 \\
\hline ClearSale & - & - & 3 & 5 & - & - & 1 & - & - & 9 \\
\hline Monsanto & 5 & - & 2 & 5 & 5 & 1 & 2 & - & 1 & 21 \\
\hline RockContent & - & 1 & 3 & 2 & 4 & - & 1 & 1 & - & 12 \\
\hline Takeda Brasil & 3 & 2 & 4 & 4 & 3 & 1 & 1 & - & - & 18 \\
\hline Mercado Livre & - & - & - & 2 & - & - & - & - & - & 2 \\
\hline Basf & 3 & - & - & 4 & 4 & 1 & - & 1 & - & 13 \\
\hline Bayer & 4 & - & 6 & 4 & 3 & 3 & 2 & - & - & 22 \\
\hline Porto Seguro & 3 & 1 & 3 & 2 & 2 & 1 & - & - & - & 12 \\
\hline CIEE & - & - & - & 2 & - & - & - & - & - & 2 \\
\hline $\begin{array}{l}\text { Dr. Emers on Lab e } \\
\text { Imagem }\end{array}$ & 3 & 1 & 2 & 2 & 2 & 1 & 1 & - & 1 & 13 \\
\hline Braskem & 4 & - & 4 & 2 & 4 & 1 & 2 & - & - & 17 \\
\hline Globosat & - & - & 1 & 1 & 3 & - & - & - & - & 5 \\
\hline GSK & 3 & - & - & 3 & 2 & - & 1 & - & - & 9 \\
\hline Amaggi & 6 & - & 7 & 3 & 4 & - & 2 & 1 & - & 23 \\
\hline Mondelez & 1 & - & 4 & 1 & 1 & 1 & 2 & - & - & 10 \\
\hline Dell & 1 & - & 2 & 4 & 2 & - & 2 & - & - & 11 \\
\hline Odebrecht & 3 & - & 4 & 1 & 4 & - & 1 & - & - & 13 \\
\hline Kimberly-Clark & 3 & - & 3 & 2 & 2 & 1 & 1 & - & - & 12 \\
\hline General Electric & 3 & - & 3 & 3 & 1 & - & 1 & - & - & 11 \\
\hline Total & 47 & 5 & 51 & 52 & 47 & 11 & 20 & 3 & 2 & 238 \\
\hline
\end{tabular}

Fonte: Elaborada pelos autores (2017).

três valores culturais, no caso confiança e honestidade, ambos na dimensão integridade, e cliente, na dimensão qualidade, superando apenas o CIEE e o Mercado Livre, que apresentaram somente dois valores culturais, cada: desenvolvimento e funcionários; e inclusão e desenvolvimento, respectivamente, todos na dimensão respeito. Daí pode-se depreender que os valores culturais divulgados pelas empresas não influenciam a preferência das mulheres no ambiente corporativo. 
Na Tabela 1, verifica-se que a dimensão respeito é a mais frequente, com 52 observações, equivalentes a 21,8\% do total possível, concentrando-se no valor cultural desenvolvimento, com 14 registros. Na sequência, ocupa a segunda posição a dimensão inovação, com 51 observações $(21,4 \%)$, concentrando 10 delas na dimensão inovação. No terceiro lugar, empataram as dimensões integridade e qualidade, com 47 ocorrências cada $(19,7 \%)$, sendo transparência e confiança os valores culturais mais frequentes na dimensão integridade, com 13 observações, e o valor cliente o mais frequente na dimensão qualidade, também com 13 registros.

Trabalho pesado foi a menos frequente das nove dimensões, com apenas duas ocorrências, sendo uma palavra citada em cada empresa, representando $0,8 \%$ das possibilidades, seguida por comunicação, com três ocorrências, sendo um registro por empresa, equivalente a 1,3\% do total; e da dimensão trabalho em equipe, com cinco observações $(2,1 \%)$.

Na pesquisa de Guiso, Sapienza e Zingales (2015), algumas empresas não anunciaram quaisquer dos valores culturais investigados, assim como outras citaram todas as categorias de valores culturais. No estudo desses autores, a inovação foi a categoria mais frequente entre as empresas, seguida por integridade e respeito.

A empresa Amaggi foi a campeã em divulgação de valores culturais, evidenciando 23 (9,7\%), distribuídos nas dimensões inovação (7), e integridade (6). A Bayer ocupou a segunda posição, registrando 22 palavras-chave $(9,2 \%)$, sendo seis delas na dimensão inovação. Em terceiro lugar, ficou a Monsanto, que registrou 21 valores (8,8\%), sendo 15 distribuídos nas dimensões integridade, respeito e qualidade, cada uma com cinco ocorrências.

A Tabela 2 apresenta a estatística descritiva dos dados coletados sobre os valores culturais, distribuídos por dimensão e por empresa.

No conjunto das empresas, observa-se uma simetria na distribuição dos valores culturais considerados, registrando-se média, moda e a mediana na faixa de 12 valores culturais. Das empresas foram registrados 238 valores culturais, já desconsideradas as ocorrências repetidas por empresa. Houve empresa que registrou somente dois valores culturais, enquanto uma delas chegou a assinalar 23. 
Tabela 2 - Estatística descritiva dos valores culturais, por empresa e por dimensão

\begin{tabular}{lcc}
\hline Variável & Por empresa & Por dimensão \\
\hline Média & 11,9 & 26,4 \\
Erro-padrão & 1,37 & 7,44 \\
Mediana & 12 & 20 \\
Moda & 12 & 47 \\
Desvio-padrão & 6,155 & 22,3277 \\
Variância & 37,8842 & 498,5277 \\
Intervalo & 21 & 50 \\
Mínimo & 2 & 2 \\
Máximo & 23 & 52 \\
Soma & 238 & 238 \\
Contagem & 20 & 9 \\
\hline
\end{tabular}

Fonte: Elaborada pelos autores (2017).

Em relação às nove dimensões de valores culturais consideradas no estudo, observa-se uma maior assimetria entre os números observados, com uma média de 26,4 valores por dimensão, apresentando maior erro-padrão e maior desvio-padrão, quando comparadas com a coluna dos valores culturais por empresa. Verifica-se que a dimensão respeito registrou 52 valores, enquanto no outro extremo a dimensão trabalho pesado assinalou apenas dois.

A Amaggi, uma das duas únicas empresas que foi constatada a participação feminina na alta administração (de apenas quatro que divulgaram a composição da diretoria), foi a que registrou o maior número de valores culturais não repetidos, totalizando 23 palavras-chave $(9,7 \%$ do total), destacando-se positivamente das demais nas dimensões inovação, com sete valores registrados, e integridade, com seis. Também com participação feminina na sua direção, a Odebrecht figurou em posição intermediária dentre todas, registrando 13 valores culturais $(5,5 \%)$, oito dos quais distribuídos entre as dimensões inovação e qualidade, com quatro em cada. Verifica-se, assim, que a dimensão inovação ocupa posição destacada nessas duas empresas.

Considerando-se as nove dimensões culturais analisadas, observa-se a seguinte ordem quantitativa decrescente de ocorrências 
de valores: respeito (52), inovação (51), integridade (47), qualidade (47), comunidade (20), segurança (11), trabalho em equipe (5), comunicação (3) e trabalho pesado (2).

\subsection{Análise dos testes estatísticos}

Para a realização dos testes estatísticos de diferenças entre médias, foram feitas duas comparações em relação à quantidade de valores culturais identificados nos websites das empresas: um teste entre aquelas empresas que apresentam algum princípio de governança corporativa e as demais; e o outro entre as empresas com reputação, ou seja, aquelas que participam de pelo menos um dos rankings reputacionais apresentados na metodologia, e as demais.

A Tabela 3 apresenta a estatística descritiva dos valores culturais entre os grupos de análise, de acordo com a apresentação (ou não) de princípios de governança corporativa.

Tabela 3 - Estatística por grupo: com e sem governança corporativa

\begin{tabular}{lllccc}
\hline & Governança & $\mathrm{N}$ & Média & Desvio Padrão & Std. Error Mean \\
\hline Valores & Com GC & 10 & 15,2 & 5,138 & 1,625 \\
Culturais & Sem GC & 10 & 8,6 & 5,42 & 1,714 \\
\hline
\end{tabular}

Fonte: Elaborada pelos autores (2017).

A Tabela 4 mostra a comparação entre as médias de valores culturais das empresas que apresentam princípios de governança corporativa e as médias das demais empresas.

Tabela 4-Comparação de médias de valores culturais entre empresas com governança corporativa e as demais

\begin{tabular}{|c|c|c|c|c|c|c|c|c|c|c|}
\hline \multirow{3}{*}{\multicolumn{2}{|c|}{$\begin{array}{c}\text { Valores Culturais } \\
\text { X } \\
\text { Governança Corporativa }\end{array}$}} & \multicolumn{2}{|c|}{ Teste de Levene } & \multicolumn{7}{|c|}{ t-test for Equality of Means } \\
\hline & & \multirow{2}{*}{$\mathrm{F}$} & \multirow{2}{*}{ Sig. } & \multirow{2}{*}{$\mathrm{t}$} & \multirow{2}{*}{ df } & \multirow{2}{*}{ Sig. } & \multirow{2}{*}{$\begin{array}{l}\text { Diferença } \\
\text { média }\end{array}$} & \multirow{2}{*}{$\begin{array}{c}\text { Erro- } \\
\text { padrão }\end{array}$} & \multicolumn{2}{|c|}{$95 \%$ de Confiança } \\
\hline & & & & & & & & & Inferior & Superior \\
\hline \multirow{2}{*}{$\begin{array}{l}\text { Valores } \\
\text { Culturais }\end{array}$} & $\begin{array}{l}\text { Igualdade de } \\
\text { variâncias } \\
\end{array}$ & 0,001 & 0,971 & 2,8 & 18 & 0,012 & 6,6 & 2,362 & 1,638 & 11,562 \\
\hline & $\begin{array}{l}\text { Não igualdade } \\
\text { de variâncias }\end{array}$ & & & 2,8 & 17,95 & 0,012 & 6,6 & 2,362 & 1,637 & 11,563 \\
\hline
\end{tabular}

Fonte: Elaborada pelos autores (2017). 
Verifica-se que a quantidade média de valores culturais das empresas que apresentam algum princípio de governança corporativa é estatisticamente superior à quantidade média de valores culturais das demais, ao nível de significância de 5\%, conforme apresentado na Tabela 4. Portanto, não se rejeita a hipótese $H_{1}$ prevista, podendo-se inferir que existe diferença estatisticamente significante em relação aos valores culturais entre empresas com indícios de adoção de princípios de governança corporativa e as demais. Esse resultado corrobora o estudo de Gorga (2004), que verificou que percepções subjetivas, refletidas na cultura e em regras informais, desempenham um papel primordial na estruturação de padrões de governança, em vez de um papel apenas residual, como tipicamente assumido.

A Tabela 5 apresenta a estatística descritiva dos valores culturais entre os grupos de análise considerando a reputação corporativa, a partir da participação das empresas em um dos seis rankings reputacionais, conforme descrito na Metodologia.

Tabela 5 - Estatística por grupo: com e sem reputação corporativa

\begin{tabular}{llcccc}
\hline & Reputação & N & Média & $\begin{array}{c}\text { Desvio } \\
\text { Padrão }\end{array}$ & $\begin{array}{c}\text { Std. Error } \\
\text { Mean }\end{array}$ \\
\hline Valores & Com Reputação & 17 & 12,41 & 6,205 & 1,505 \\
Culturais & Sem Reputação & 3 & 9 & 6,083 & 3,512 \\
\hline
\end{tabular}

Fonte: Elaborada pelos autores (2017).

Na Tabela 6 são evidenciados os resultados do teste de diferença de médias de valores culturais entre as empresas com reputação corporativa e as demais.

Tabela 6 - Comparação de médias de valores culturais entre as empresas com reputação corporativa e as demais

\begin{tabular}{|c|c|c|c|c|c|c|c|c|c|c|}
\hline \multirow{3}{*}{\multicolumn{2}{|c|}{$\begin{array}{c}\text { Valores Culturais } \\
\mathrm{X} \\
\text { Reputação Corporativa }\end{array}$}} & \multicolumn{2}{|c|}{ Teste de Levene } & \multicolumn{7}{|c|}{ t-test for Equality of Means } \\
\hline & & \multirow{2}{*}{$\mathrm{F}$} & \multirow{2}{*}{ Sig. } & \multirow{2}{*}{$\mathrm{t}$} & \multirow{2}{*}{ df } & \multirow{2}{*}{ Sig. } & \multirow{2}{*}{$\begin{array}{c}\text { Diferença } \\
\text { média }\end{array}$} & \multirow{2}{*}{$\begin{array}{l}\text { Erro- } \\
\text { padrão }\end{array}$} & \multicolumn{2}{|c|}{$95 \%$ de Confiança } \\
\hline & & & & & & & & & Inferior & Superior \\
\hline \multirow{2}{*}{$\begin{array}{l}\text { Valores } \\
\text { Culturais }\end{array}$} & $\begin{array}{l}\text { Igualdade de } \\
\text { variâncias }\end{array}$ & \multirow[t]{2}{*}{0,001} & \multirow[t]{2}{*}{0,982} & 0,9 & 18 & 0,391 & 3,41 & 3,878 & $-4,735$ & 11,558 \\
\hline & $\begin{array}{l}\text { Não igualdade } \\
\text { de variâncias }\end{array}$ & & & 0,9 & 2,79 & 0,442 & 3,41 & 3,821 & $-9,281$ & 16,105 \\
\hline
\end{tabular}

Fonte: Elaborada pelos autores (2017). 
Como mostra as Tabelas 5 e 6, na comparação entre as empresas com reputação corporativa e as demais, o resultado do teste revela que a quantidade média de valores culturais das empresas com reputação não é estatisticamente diferente da quantidade média de valores culturais daquelas sem reputação corporativa, não sendo possível constatar diferença entre os dois grupos. Rejeita-se, assim, a hipótese $\mathrm{H}_{2}$, de que há diferença entre as médias dos dois grupos. Portanto não se pode dizer que existe diferença estatisticamente significante em relação aos valores culturais entre as empresas com reputação e as demais.

Esse resultado vai de encontro ao achado por Caixeta et al. (2011), que explicam que a reputação está relacionada com a cultura, refletindo as relações entre os administradores e os principais stakeholders da empresa, visto que os valores culturais não apenas definem o significado da empresa, mas, adicionalmente, guiam e justificam as estratégias dos administradores para lidar com os diversos públicos.

Adicionalmente, com os dados das empresas, foi possível realizar o teste de correlação de Pearson entre as variáveis valores culturais, governança corporativa e reputação corporativa. A Tabela 7 mostra o resultado do teste de correlação de Pearson.

Tabela 7 - Correlação entre valores culturais, governança corporativa e reputação corporativa

\begin{tabular}{l|l|c|c|c}
\hline \multicolumn{2}{l|}{} & Valores Culturais & Reputação & Governança \\
\hline \multirow{3}{*}{ Valores Culturais } & Pearson Correlation & 1 & 0,203 &, $550^{*}$ \\
& Sig. & & 0,391 & 0,012 \\
& $\mathrm{~N}$ & 20 & 20 & 20 \\
\hline \multirow{5}{*}{ Reputação } & Pearson Correlation & & 1 & 0,14 \\
& Sig. & & & 0,556 \\
& $\mathrm{~N}$ & & 20 & 20 \\
\hline \multirow{3}{*}{ Governança } & Pearson Correlation & & & 1 \\
& Sig. & & & 20 \\
\hline
\end{tabular}

Fonte: Elaborada pelos autores (2017). 
Na Tabela 7, observa-se que há correlação ou interdependência entre os valores culturais das empresas e os princípios de governança corporativa por elas apresentados, ao nível de significância de 5\% (p-value: 0,012). Essa correlação corrobora o estudo de Lopes, Valentim e Fadel (2014) ao relacionar tais variáveis e verificar que a governança corporativa requer muitas vezes uma mudança cultural, que necessita reforçar os comportamentos culturais de seus membros, com apoio em princípios e valores que incrementam a relação entre a organização e seus stakeholders. De forma similar, Rodrigues e Pérez (2016) também fundamentam essa correlação, na medida em que, apoiados pela Teoria Institucional, afirmam que as organizações estão continuamente adaptando suas estruturas de governança e políticas institucionais ao contexto cultural em que operam.

Não se pode afirmar o mesmo entre as variáveis valores culturais e reputação corporativa, pois, como mostra a Tabela 7, não existe correlação estatisticamente significante entre tais variáveis, com nível de significância superior a 10\% (p-value: 0,391). Do mesmo modo, é possível verificar também a inexistência de correlação estatisticamente significante entre as variáveis reputação e governança corporativa (p-value: 0,556$)$.

\section{CONSIDERAÇões FINAIS}

O estudo teve origem na curiosidade de se investigar os valores culturais organizacionais que atraem as mulheres profissionais nas empresas brasileiras, a partir do levantamento de dados e informações das 20 empresas componentes do ranking divulgado pela comunidade de carreiras Love Mondays em 2017, formado pela percepção das mulheres que as elegeram como suas preferidas para trabalhar. A pesquisa apontou que, nos últimos dois anos, a cultura da empresa se destaca em primeiro lugar entre os fatores que atraem as mulheres.

Considerando-se que a cultura, sozinha, não transmite todas as características das empresas, instigou-se analisar, conjuntamente, outras variáveis, a fim de se fazer possíveis comparações e identificar importantes associações e correlações entre elas. Assim, foram analisadas a participação feminina na alta administração, a governança corporativa e a reputação corporativa. 
Os resultados descritivos mostraram que a dimensão cultural respeito foi a mais presente nas empresas, com 21,8\% das observações totais, concentrando-se no valor cultural desenvolvimento, que foi a palavra-chave mais frequentemente observada, por 14 empresas. E a dimensão trabalho pesado foi a menos expressiva, sendo citada por apenas duas empresas. Observou-se, ainda, que a quantidade de valores culturais presentes nas empresas não se correlaciona com a preferência das mulheres profissionais por trabalhar nessas empresas.

Os testes de comparação entre médias mostraram que as empresas adeptas de princípios de governança corporativa registram uma quantidade média de valores culturais superior à das demais, refutando a hipótese $\mathrm{H}_{1}$, ou seja, há diferença entre os grupos. Diferentemente, no teste que comparou as médias entre o grupo de empresas com reputação corporativa e as demais, o resultado revelou não haver diferenças entre os grupos, não apresentando suporte para rejeitar a hipótese $\mathrm{H}_{2}$.

Por fim, o teste de correlação de Pearson entre as variáveis valores culturais e governança corporativa mostrou haver correlação significante entre tais variáveis. Já entre os valores culturais e a reputação, o teste não apresentou associação significante entre as variáveis, sugerindo a não existência de correlação. Entre as variáveis reputação e governança corporativa, o teste também demonstrou que não há relação significativa, sugerindo a ausência de correlação.

Vale lembrar que as práticas de governança corporativa são importantes instrumentos de transparência, que ao relacionar-se com os valores culturais e a reputação, podem aumentar a credibilidade por parte dos stakeholders, maximizando características empresariais como a de socialmente responsável.

Do exposto, os resultados do estudo contribuem para uma melhor compreensão da relação entre os valores culturais e a reputação e a governança corporativas, objetivando à expansão do conhecimento e base para novos estudos. Cabe ressaltar que não foi possível comparar detalhadamente esses resultados com achados empíricos anteriores, pois não foram encontrados estudos que analisaram as temáticas aqui estudadas, em conjunto, e, em especial, considerando a participação e percepção das mulheres no ambiente corporativo. 
Avalia-se que o estudo alcançou seus objetivos, e que conseguiu responder ao problema de pesquisa formulado, assim como contribui para enriquecer a literatura científica no que tange à associação das variáveis analisadas (valores culturais, reputação e governança corporativas), aplicando-se método empírico para reforçar as análises, unindo os aspectos qualitativo e quantitativo para o aprimoramento da ciência. Dessa forma, reforça-se a importância da cultura organizacional como tema de interesse, porém, como encontrado, não se pode afirmar que é elemento decisivo para motivar a preferência de mulheres quanto ao ambiente corporativo para trabalhar.

Dentre as limitações do estudo, destacou-se a impossibilidade de se estabelecer outras análises sobre a participação das mulheres na administração das empresas, haja vista que das 20 empresas, apenas quatro divulgaram a composição da alta administração, aí incluída a diretoria executiva, sendo que a participação feminina na alta direção foi registrada em apenas duas delas. Outra limitação refere-se à baixa quantidade de informações disponíveis nos websites das empresas, que, por serem em sua maioria sociedades anônimas de capital fechado e sociedades empresárias limitadas, não seguem um padrão de divulgação de informações voltadas para o público externo.

Sugere-se ampliar as análises realizadas no presente estudo, para uma população com maior número de empresas, de diferentes setores e com dados e informações públicas disponíveis para fundamentar as análises. Além disso, recomenda-se relacionar as variáveis deste estudo com construtos econômico-financeiro como o desempenho e a criação de valor corporativos.

\section{REFERÊNCIAS}

ADAMS, R.B.; FERREIRA, D. Women in the boardroom and their impact on governance and performance. Journal of financial Economics, v. 94, n. 2, p. 291-309, 2009.

ANDRADE, A.; ROSSETTI, J. P. Governança corporativa: fundamentos, desenvolvimento e tendências. 4. ed. São Paulo: Atlas, 2009.

BANDEIRA, M. T. S. S.; GÓIS, A. D.; DE LUCA, M. M. M.; VASCONCELOS, A. C. Reputação corporativa negativa e o desempenho empresarial. Revista de Contabilidade e Organizações, v. 24, n. 9, p. 72-83, 2015. 
CAIXETA, C. G. F; LOPES, H. E. G.; BERNARDES, P.; CARDOSO, M. B. R.; CARVALHO NETO, A. M. Reputação corporativa e desempenho econômico-financeiro: um estudo em cinco grandes grupos empresariais brasileiros. Revista Eletrônica de Gestão Organizacional, v. 1, n. 9, p. 86-109, 2011.

CALEGARI, I. P.; GONÇALVES, R. S.; SERRANO, A. L. M.; RODRIGUES, J. M. Efeitos da reputação corporativa no custo de capital próprio em empresas brasileiras listadas. Revista Universo Contábil, v. 12, n. 1, p. 178-98, 2016.

CAMERON, K. S.; QUINN, R. E. Diagnosing and changing organizational culture. San Francisco: Jossey-Bass, 2006.

CASAVECHIA, C.; MEDEIROS, C. R. de O.; VALADÃO JÚNIOR, V. M. Manifestações culturais na perspectiva da integração: construindo um holograma. Revista Eletrônica Gestão e Sociedade, v. 5, n. 12, p. 216-35, 2011.

CAVAZOTTE, F. S. C. N.; OLIVEIRA, L. B.; MIRANDA, L. C. Desigualdade de gênero no trabalho: reflexos nas atitudes das mulheres e em sua intenção de deixar a empresa. Revista de Administração, v. 45, n. 1, p. 70-83, 2010.

CERIBELI, H. B.; CERIBELI, M. C. B.; FERREIRA, F. J. R. Análise da qualidade de vida no trabalho sob a perspectiva das diferenças entre gêneros. Reuna, v. 21, n. 3, p. 5-24, 2016.

CHUN, R. Corporate reputation: meaning and measurement. International Journal of Management Reviews, v. 7, n. 2, p. 91-109, 2005.

DEMO, G.; FERNANDES, T.; FOGAÇA, N. A Influência dos Valores Organizacionais na Percepção de Políticas e Práticas de Gestão de Pessoas. REAd. Revista Eletrônica de Administração, v. 23, n. 1, p. 89-117, 2017.

DUTRA, R. S.; PARENTE, P. H. N. A Governança Corporativa e Desempenho Empresarial: uma análise sob o enfoque da reputação corporativa das empresas brasileiras. RAGC - Revista de Auditoria Governança e Contabilidade, v. 6, n. 26, p. 47-63, 2018.

FÁVERO, L. P. Manual de análise de dados. 1. ed. Rio de Janeiro: Elsevier, 2017.

FIELD, A. Descobrindo a estatística usando o SPSS. Porto Alegre: Artmed, 2009.

FELDMAN, P. M.; BAHAMONDE, R. A.; BELLIDO, I. V. A new approach for measuring corporate reputation. Revista de Administração de Empresas, v. 54, n. 1, p. 53-66, 2014.

FERREIRA, R. M.; LIMA, S. L. L.; GOMES, A. R. V.; MELLO, G. R. Governança Corporativa: Um Estudo Bibliométrico da Produção Científica entre 2010 a 2016. Revista Organizações em Contexto, v. 15, n. 29, p. 323-42, 2019.

FLAMHOLTZ, E. G.; RANDLE, Y. Corporate culture, business models, competitive advantage, strategic assets and the bottom line: theoretical and measurement issues. Journal of Human Resource Costing \& Accounting, v. 16, n. 2, p. 76-94, 2012.

FLATT, S.; KOWALCZYK, S. Creating competitive advantage through intangible assets: the direct and indirect effects of corporate culture and reputation. Advances in Competitiveness Research, v. 16, n. 1, p. 13-30, 2008. 
FLEURY, M. T. Estórias, mitos, heróis - cultura organizacional e relações de trabalho. Revista de Administração de Empresas, v. 27, n. 4, p.7-18, 1987.

FLEURY, M. T.; FISCHER, R. M. Cultura e poder nas organizações. São Paulo: Atlas,1989.

FREITAS, M. E. Cultura organizacional: grandes temas em debate. Revista de Administração de Empresas, v. 31, n. 3, p. 73-82, 1991.

GORGA, Érica. A cultura brasileira como fator determinante na governança corporativa e no desenvolvimento do mercado de capitais. Revista de Administração, v. 39, n. 4, p. 30926, 2004.

GREYSER, S. A. Advancing and enhancing corporate reputation. Corporate Communications: An International Journal, v. 4, n. 4, p. 177-81, 1999.

GUISO, L.; SAPIENZA, P.; ZINGALES, L. The value of corporate culture. Journal of Financial Economics, v. 117, p. 60-75, 2015.

HARTNELL, C.A.; OU, A. Y.; KINICKI, A. Organizational culture and organizational effectiveness: A meta-analytic investigation of the competing values framework's theoretical suppositions. Journal of Applied Psychology, v. 96, n. 4, p. 677-94, 2011.

HOOBLER, J. M.; MASTERSON, C. R.; NKOMO, S. M.; MICHEL, E. J. The Business Case for Women Leaders: Meta-Analysis, Research Critique, and Path Forward. Journal of Management, v. 44, n. 6, p. 2473-99, 2016.

IBGC. Instituto Brasileiro de Governança Corporativa. Código das melhores práticas de governança corporativa. 5. ed. São Paulo: IBGC, 2015.

JENSEN, M. C.; MECKLING, W. C. Theory of the firm: managerial behaviour, agency costs and ownership structure. Journal of Financial Economics, v. 3, n. 4, p. 305-60, 1976.

JURKUS, A. F.; PARK, J. C.; WOODARD, L. S. Women in top management and agency costs. Journal of Business Research, v. 64, n. 2, p. 180-6, 2011.

LEE, J.; ROH, J. J. Revisiting corporate reputation and firm performance link. Benchmarking: An International Journal, v. 19, n. 4/5, p. 649-64, 2012.

LIRA, S. A.; CHAVES NETO, A. Coeficientes de correlação para variáveis ordinais e dicotômicas derivados do coeficiente linear de Pearson. Recie, v. 15, n. 1/2, p. 45-53, 2006.

LIU, Y.; WEI, Z.; XIE, F. Do women directors improve firm performance in China? Journal of Corporate Finance, v. 28, p. 169-84, 2014.

LOPES, E. C.; VALENTIM, M. P.; FADEL, B. Efeitos da cultura organizacional no desenvolvimento dos modelos de governança corporativa. Revista Famecos, v. 21, n. 1, p. 268-86, 2014.

LOVE MONDAYS. As 20 empresas mais bem avaliadas pelas mulheres, 2017. Disponível em: $<$ https://www.lovemondays.com.br/setores-e-empresas-mais-bem-avaliados-pelas-mulheres $>$. Acesso em: 4 maio 2017.

LOVE MONDAYS. Sobre o Love Mondays, 2019. Disponível em: $<$ https://www.lovemondays. com.br/sobre>. Acesso em: 30 mar. 2019. 
MACEDO, M. A. S.; CORRAR, L. J. A. Análise comparativa do desempenho contábil-financeiro de empresas com boas práticas de governança corporativa no Brasil. Revista Contabilidade e Controladoria, v. 4, n. 1, p. 42-61, 2012.

MALHOTRA, N. Pesquisa de marketing. 3. ed. Porto Alegre: Bookman, 2001.

MENEZES, R. S. S.; OLIVEIRA, J. L.; DINIZ, A. P. R. Simbolismos de gênero e gestão: uma análise das feminilidades de executivas. Revista de Gestão e Secretariado, v. 4, n. 1, p. 1-22, 2013.

MILLS, A. J. Organization, gender and culture. Organization Studies, v. 9, n. 3, p. 351-69, 1988.

MINADEO, R. Visão Geral sobre Governança Corporativa e Análises de Situações Empresariais Críticas à Luz de seus Eixos Centrais. Revista Organizações em Contexto, v. 15, n. 29, p. 39-67, 2019.

NASCIMENTO, S. D. Precarização do trabalho feminino: a realidade das mulheres no mundo do trabalho. Revista de Políticas Públicas, n. Esp., p. 339-46, 2016.

NARDI, P. C. C.; NAKAO, S. H. Impacto da entrada nos níveis diferenciados de governança corporativa sobre a imagem institucional das empresas. Revista Contabilidade Vista e Revista, v. 19, n. 2, p. 85-111, 2008.

NUNES, T. S.; TOLFO, S. R.; GARCIA, I. S. Valores Organizacionais Declarados e Praticados na Universidade Federal de Santa Catarina. Administração Pública e Gestão Social, v. 10, n. 2, p. 123-35, 2018.

OLIVEIRA, I. G. S.; CARDOSO, S. P.; DIAS, R. S.; BORGES JUNIOR, P. Influence of Female Boardroom Presence on Firm Value: An Analysis on Companies Listed on B3. Revista de Negócios, v. 23, n. 3, p. 49-57, 2018.

OLIVEIRA, A. R.; GAIO, L. E.; BONACIM, C. A. G. Relações de gênero e ascensão feminina no ambiente organizacional: um ensaio teórico. Revista de Administração da Universidade Federal de Santa Maria, v. 2, n. 1, p. 80-97, 2009.

PAULA, P.; STEFANO, S. R.; ANDRADE, S. M.; ZAMPER, M. A. Clima e cultura organizacional em uma organização pública. Gestão \& Regionalidade, v. 27, n. 81, p. 59-73, 2011.

PETTIGREW, A. M. On Studying Organizational Cultures. Administrative Science Quarterly, v. 2, n. 4, 570-81, 1979.

PIRES, J. C. S.; MACEDO, K. B. Cultura organizacional em organizações públicas no Brasil. Revista de Administração Pública, v. 40, n. 1, p. 81-105, 2006.

POUND, J. The promise of the governed corporation. Harvard Business Review on Corporate Governance, From the march-april issue. p. 79-104, 1995.

RINDOVA, V. P.; WILLIAMSON, I. O.; PETKOVA, A. P. Being good or being known: an empirical examination of the dimensions, antecedents, and consequences of organizational reputation. Academy of Management Journal, v. 48, n. 6, p. 1033-49, 2005.

ROBERTS, P. W.; DOWLING, G. R. Corporate reputation and sustained superior financial performance. Strategic Management Journal, v. 23, n. 12, p.1077-93, 2002.

RODRIGUES, M. M. M.; PÉREZ, B. E. Does the institutional environment affect CSR disclosure? The role of governance. Revista de Administração de Empresas, v.56, n.6, p. 641-54, 2016. 
SALVAGNI, J.; CANABARRO, J. Mulheres líderes: as desigualdades de gênero, carreira e família nas organizações de trabalho. Revista de Gestão e Secretariado, v. 6, n. 2, p. 88-110, 2015. SHLEIFER, A.; VISHNY, R. A survey of corporate governance. Journal of Finance, v. 52, n. 2, p. 737-83, 1997.

SILVA JÚNIOR, R. R. da.; JUNQUEIRA, L. R.; BERTUCCI, L. A. A relação entre a adoção das práticas de governança corporativa e a alavancagem financeira das empresas brasileiras do setor energético no ano de 2008. Revista Eletrônica Gestão e Sociedade, v. 3 n. 6, p. 315-34, 2009.

TOMÉ, A. S.; VIEIRA, A. M. Cultura organizacional e formação identitária: um estudo com gerentes do setor bancário. Caderno de Administração, v. 25, n. 2, p. 44-57, 2017.

VASCONCELOS, K. C. A.; MERHI, D. Q.; SILVA JÚNIOR, A.; SILVA, P. O. M. Cultura, liderança e compartilhamento do conhecimento organizacional. Revista de Administração FACES Journal, v. 12, n. 1, p. 13-31, 2013.

VIEIRA, A. M. Cultura organizacional em instituições de ensino: mapeamento e análise descritivo-interpretativa da produção acadêmica (1990-2005). 2007. 237f. Tese (Doutorado em Educação) - Universidade Estadual Paulista (UNESP), Marília, 2007.

XU, X.; LI, Y.; CHANG, M. Female CFOs and loan contracting: financial conservatism or gender discrimination? An empirical test based on collateral clauses. China Journal of Accounting Research, v. 9, n. 2, p. 153-73, 2016.

Recebido em: 10-4-2019

Aprovado em: 24-10-2019

Avaliado pelo sistema double blind review.

Editor: Coordenação do PPGA/UMESP

Disponível em http://mjs.metodista.br/index.php/roc 